Published in final edited form as:

Proc IEEE Int Symp Biomed Imaging. 2013 December 31; 2013: 1252-1255. doi:10.1109/ISBI. 2013.6556758.

\title{
LOCALLY WEIGHTED TOTAL VARIATION DENOISING FOR RINGING ARTIFACT SUPPRESSION IN PET RECONSTRUCTION USING PSF MODELING
}

\author{
Arthur Mikhno ${ }^{1}$, Elsa D. Angelini ${ }^{1,3}$, Bing Bai ${ }^{2}$, and Andrew F. Laine ${ }^{1}$ \\ ${ }^{1}$ Department of Biomedical Engineering, Columbia University \\ ${ }^{2}$ Signal and Image Processing Institute, University of Southern California \\ ${ }^{3}$ Institut Mines-Telecom, Telecom ParisTech, CNRS LTCI, Paris, France
}

\begin{abstract}
Iterative reconstruction with point spread function (PSF) modeling improves contrast recovery in positron emission tomography (PET) images, but also introduces ringing artifacts and over enhancement that is contrast and object size dependent. Mitigation of these artifacts is crucial for clinical and research purposes. In this work we introduce a new iterative regularized reconstruction method that incorporates locally-weighted total variation denoising designed to suppress artifacts induced by PSF modeling. The reconstruction method is evaluated on a simulated cylindrical phantom and preliminary results show that ringing artifacts are suppressed while contrast recovery is maintained.
\end{abstract}

\section{Index Terms}

PET; image reconstruction; point spread function; MLEM; Total Variation

\section{INTRODUCTION}

Existing and commonly used iterative reconstruction techniques in positron emission tomography (PET) provide a flexible framework for modeling the physics and the scanner geometry, yielding greater image contrast, visual quality and noise robustness than analytical reconstruction methods. Modeling and accounting for the physics of the emissions (e.g. positron range) and the detection processes (e.g. crystal scattering or depth of interaction) is possible by measuring and incorporating the detector point spread function (PSF) into the reconstruction process. Such approach has been shown shown to improve spatial resolution and image contrast [1]. Unfortunately, it also introduces significant edge artifacts such as over enhancement and Gibbs type of ringing that are both contrast and size dependent (leading to up to $70 \%$ overshoot in a cylinder phantom) [2, 3]. Mitigation of the above artifacts is crucial to ensure image quantification accuracy, especially since reconstruction with PSF modeling is now implemented and widely used in clinical PET/CT systems.

Some work has been done to characterize and compensate for PSF modeling artifacts. Bai et al. Showed that the overshoot depends on region sizes and contrast ratios [2]. Snyder et al. observed that the overshoot might be explained by the mismatch between the true and measured PSF [4]. Tong et al. reported that ringing frequency and amplitude are related to 
objects' sizes [5]. A number of different mitigation strategies have been discussed, such as under sampling the PSF and post-filtering the reconstructed images [5]. While these approaches reduce artifacts, they also blur the PET images and undermine the benefits of PSF modeling [4]. Rapisarda et al. incorporated a new regularization prior into the reconstruction process that locally modifies the image estimate at each iteration in an attempt to locally control edge enhancement [6]. This method is promising but currently requires optimizing two parameters and while artifacts are suppressed there is loss of contrast recovery.

Total variation (TV) denoising methods have been adapted for Bayesian iterative reconstruction algorithms suitable for use with PET, showing effective suppression of noise and reconstruction of homogenous regions with sharp edges [7]. Previous works assumed a uniform distribution of noise and therefore applied TV globally, which is not suitable for localized PSF modeling artifacts. In this work we develop a new locally-weighted TV strategy, where denoising weights are derived empirically from the data and are incorporated directly into the iterative reconstruction process. We evaluated the proposed reconstruction method on a simulated cylindrical phantom image, assessing contrast and resolution recovery.

\section{METHODS}

\subsection{PET iterative image reconstruction: MLEM algorithm}

The maximum likelihood estimate of the PET images is computed using the maximum likelihood expectation maximization (MLEM) algorithm [5]. Following the notation from [6], the MLEM iterative update equation is given as follows:

$$
\lambda_{b}^{k+1}=\frac{\lambda_{b}^{k}}{B P_{b} 1} B P_{b} \frac{y_{d}}{P_{d} \lambda_{b^{\prime}}^{k}}=\lambda_{b}^{k} u_{b}^{k}
$$

where $\lambda_{b}^{k}$ represents the counts in a voxel $b$ within the image $\lambda$ at iteration $k, y_{d}$ is the measured projection recorded as number of counts along the line of response (LOR) $d$, and $\mathbf{1}$ is a unit matrix of the same size as $y_{d}$. The forward $P_{d}(\cdot)_{b}=\sum[(\cdot)]_{b} p_{\mathrm{bd}}$ and backward $B P_{b}(\cdot)_{d}=\sum[(\cdot)]_{d} p_{\text {bd }}$ projectors contain a weight matrix $p_{b d}$ that links the voxel $b$ and LOR $d$. The term $B P_{b} 1$ is called the geometric sensitivity, and can be pre-computed prior to reconstruction. Projection terms applied at iteration $k$ can be combined into a multiplicative updating term $u_{b}^{k}$.

\subsection{PSF modeling}

Following the methodology introduced by Rapisardra et al., incorporating the PSF model into (1) is achieved by modifying the projectors as follows:

$$
\begin{aligned}
P_{d}(\cdot)_{b} & =\sum[(\cdot) * \mathrm{PSF}]_{b} p_{\mathrm{bd}} \\
B P_{b}(\cdot)_{d} & =\mathrm{PSF}^{T} * \sum[(\cdot)]_{d} p_{\mathrm{bd}}
\end{aligned}
$$

where $P S F$ represents the PSF kernel and * is a discretized convolution operator as defined in Appendix of [6]. For a symmetric kernel, $P S F=P S F^{T}$. MLEM reconstruction that utilizes Eq. (2)-(3) will be here referred to as PSF-MLEM. 


\subsection{Reconstruction software and simulated data}

All reconstructions were performed using the STIR open source C++ software (v2.2) [9]. The modified projectors in Eq. (2) and (3) were written in C++ within STIR. A $200 \mathrm{~mm}$ diameter cylindrical phantom object was simulated, with a background intensity equal to 10 , three hot spots of diameter $25 \mathrm{~mm}, 16 \mathrm{~mm}, 12 \mathrm{~mm}$ with a $1.5: 1$ contrast ratio (CR), and three $8 \mathrm{~mm}$ diameter hot spots with CR of 1.25:1, 1.5:1 and 2:1, respectively. The phantom was blurred with a symmetrical $4.5 \mathrm{~mm}$ FWHM Gaussian kernel [10] to simulate the resolution loss due to physics inherent in the ECAT HR+ scanner (Siemens/CTI) at use in our facility. Figure 1 illustrates the phantom image with resolution and contrast loss due to blurring.

\subsection{Total Variation denoising}

Following the notations above, at each iteration of Eq. (1) the total variation problem amounts to finding an estimate image $\lambda^{\hat{k}}$ that satisfies the following optimization problem:

$$
\widehat{\lambda}^{k}=\min \frac{1}{2}\left\|\lambda^{k}-\widehat{\lambda}^{k}\right\|^{2}+\beta\left|\nabla \widehat{\lambda}^{k}\right|
$$

where $|\cdot|$ and $\|\cdot\|$ are the $L_{1}$ and $L_{2}$ norms, respectively and $\beta$ is the regularization weight. TV optimization was performed using the toolbox [7] implemented in Matlab ${ }^{1}$.

\subsection{Locally-weighted Total Variation denoising}

The classical framework given by Eq. (4) minimizes TV over the whole image, while PSF modeling introduces local artifacts. We therefore propose to locally integrate the TV filtered estimate $\lambda^{\hat{k}}$ into $\lambda_{b}^{k}$, re-expressing $\lambda^{\hat{k}}$ as:

$$
\widehat{\lambda}_{b}^{k}=\lambda_{b}^{k}+\left(\widehat{\lambda}_{b}^{k}-\lambda_{b}^{k}\right)=\lambda_{b}^{k}+\Delta \widehat{\lambda}_{b}^{k}
$$

where, $\Delta \hat{\lambda}_{b}^{k}=\hat{\lambda}_{b}^{k}-\lambda_{b}^{k}$ represents the net change in each voxel $b$ on the image estimation after TV denoising. Since TV filtering is only needed at specific voxel locations we propose to locally constrain TV enforcement by introducing a local weight on each TV filtered voxel, defining:

$$
\dot{\lambda}_{b}^{k}=\lambda_{b}^{k}+w_{b} \Delta \hat{\lambda}_{b}^{k}
$$

where $\dot{\lambda}_{b}^{k}$ is the locally weighted TV estimate and $w_{b}$ is a spatially-varying weight imposed on the net change of each voxel. Note that if $w_{b}=1$ then $\lambda_{b}=\lambda_{b}$, corresponding to the classical solution to Eq. (4). The PSF-MLEM with TV denoising (TV-PSF-MLEM) algorithm is thus given as follows:

For iteration 1 to $\mathrm{N}$ :

Step 1: obtain $\lambda_{b}^{k+1}$ from $\lambda_{b}^{k}$ using Eq. (1)

Step 2: apply Eq. (4) on $\lambda_{b}^{k+1}$ to obtain $\hat{\lambda}_{b}^{k+1}$

Step 3: apply Eq. (6) on $\hat{\lambda}_{b}^{k+1}$ to obtain $\dot{\lambda}_{b}^{k+1}$

Step 4: repeat from Step 1 using $\lambda_{b}^{k}=\dot{\lambda}_{b}^{k+1}$

\footnotetext{
${ }^{1}$ Tremoulheac, Benjamin (2012). Split Bregman method for Total Variation Denoising (http://www.mathworks.com/matlabcentral/ fileexchange/36278), MATLAB Central File Exchange. Retrieved September 29, 2012
} 


\subsection{Definition of the TV-denoising spatial weights}

We needed to design a local weighting scheme such that flat homogenous regions and edges were preserved from the original MLEM estimation, while edge ringing was reduced with TV filtering. Instead of filtering the current estimate relying on its edge maps, we chose a novel approach, to see if we could exploit the information learned on the evolution of $\lambda^{k}$ over the iterations of the MLEM reconstruction. Figure 2 shows, on the phantom's horizontal midline profile, the evolution of $\lambda^{k}$ over several MLEM iterations (toward convergence), as well as the number of iterations required for each voxel along the profile to converge and the second spatial derivative of the MLEM profile at convergence.

We based our weighting strategy on a few observations: (a) each cylinder's edges have inflection points (defined as zero-crossing of the second spatial derivative) that spatially converge very quickly, (b) the interiors of flat regions converge quickly, while edge refinement continues for a long time (as the peak expands while the support shrinks); (c) the convergence rate for the $8 \mathrm{~mm}$ cylinders is contrast dependent, and faster for the cylinder with CR 1.25:1 versus 1.5:1, and (d) while the rate of convergence globally mimics the second derivative of the reconstructed profile, there are many local differences. This suggests that there might be unique information contained in the evolution of the MLEM reconstructions that cannot be derived directly from the structure of the reconstructed image.

Based on these observations we designed a new spatial weight $w_{b}$ as follows:

$$
\begin{aligned}
& c_{b}=\min _{k} \operatorname{abs}\left(1-u_{b}^{k}\right) \leq 1 e^{-4} \\
& w_{b}=1-\frac{c_{b}-\min \left(c_{b}\right)}{\max \left(c_{b}-\min \left(c_{b}\right)\right)}
\end{aligned}
$$

where $c_{b}$ is defined as the earliest MLEM iteration $k$ in which voxel $b$ converges (in practice when $u_{b}^{k} \simeq 1$ ), and $w_{b}$ is derived by normalizing $c_{b}$ such that $0 \leq w_{b} \leq 1$. Figure 3 illustrates the obtained spatial TV-weights $w_{b}$.

\section{RESULTS}

\subsection{Evaluation setup on synthetic phantom data}

To test whether our spatially weighted TV denoising approach improved image quality, we ran TV-PSF-MLEM empirically setting $\beta=0.02$. Over several experiments with $\beta=\{.005$, . $01, .02, .04\}$ we found that $\beta=0.02$ yielded optimal ringing suppression without degrading image quality. For the MLEM reconstruction, we initialized with $\lambda^{0}=1$. The other reconstructions were initialized with the MLEM estimate.

We quantitatively evaluated contrast recovery using the recovery coefficient (RC) measure, defined as:

$$
\mathrm{RC}=\sum_{b \in \Omega_{\mathrm{ROI}}} \lambda^{\text {recon }} / \sum_{b \in \Omega_{\mathrm{ROI}}} \lambda_{b}^{\text {true }}
$$

where the $\Omega_{R O I}$ is the region of interest (e.g. inside a cylinder), $\lambda^{\text {recon }}$ is the reconstruction being evaluated and $\lambda^{\text {true }}$ is the original non-blurred (ideal) phantom. RC measures can be above or below one and $\mathrm{RC}=1$ for a perfect reconstruction.

The synthetic cylindrical phantom was reconstructed (200 iterations) with the three different algorithms (MLEM, PSF-MLEM and TV-PSF-MLEM) and the RC values were measured inside each six cylinders. TV-PSF-MLEM yielded better RC measures than MLEM, and only slightly lower than PSF-MLEM, in all cylinders (Figure 4). 


\subsection{Evaluation of ringing artifacts at edges}

To evaluate ringing artifacts, we visually compared the resulting images. For the PSFMLEM reconstruction, results, illustrated in Figure 5, show that MLEM had no ringing artifact (as expected), PSF-MLEM generates ringing in the background and at edges of the $25 \mathrm{~mm}$ and $16 \mathrm{~mm}$ cylinders, as well as over enhancement of the $12 \mathrm{~mm}$ cylinder. For the $8 \mathrm{~mm}$ cylinders there were contrast-dependent over- and under- enhancement.

For the TV-PSF-MLEM reconstruction, results, illustrated in Figure 6, show that ringing artifacts were suppressed on all cylinders edges. The contrast in the $25 \mathrm{~mm}$ and $16 \mathrm{~mm}$ cylinders was very well recovered, and over-estimation of the $12 \mathrm{~mm}$ cylinder intensity was reduced, compared to PSF-MLEM reconstruction (cf. Figure 5). The contrast in the $8 \mathrm{~mm}$ cylinders was similar for TV-PSF-MLEM and PSF-MLEM reconstructions, demonstrating that locally-weighted TV denoising can suppress ringing within large structures while maintaining the contrast in small regions. In addition to removing ringing artifacts, TV also enforces homogeneity in large regions. This effect is illustrated in Figure 7 in the $25 \mathrm{~mm}$ cylinder, where MLEM yielded a noisy image, PSF-MLEM induced heterogeneity and ringing at the edge, while TV-PSF-MLEM prevented the ringing and enforced homogeneity inside the cylinder.

\section{CONCLUSIONS}

We introduced TV denoising in the PSF-MLEM iterative reconstruction method for PET images. This method was evaluated on a synthetic phantom image with multiple cylinders varying in size and contrast. Results showed that TV-PSF-MLEM maintains the good contrast recovery properties of PSF-MLEM while reducing ringing artifacts at edges.

These results are promising, but remain preliminary and need to be investigated further on more complex synthetic phantoms. Indeed, the proposed reconstruction method needs to be evaluated in objects with greater variations in size and contrast and including negative contrast (i.e. cold spots). Robustness with respect to the presence of Poisson noise in the sinogram should also be evaluated. Regarding the methodology itself, the influence of the TV parameter $\beta$ needs to be studied, and we need to derive stopping criterions of the reconstruction process optimized separately for each of the reconstruction approaches, instead of using the same fixed number of iterations. Finally, further characterization of the proposed reconstruction method using a physical phantom will be the subject of future work.

\section{References}

1. Sureau FC, et al. Impact of Image-Space Resolution Modeling for Studies with the High-Resolution Research Tomograph. Journal of Nuclear Medicine. May; 2008 49(6):1000-1008. [PubMed: 18511844]

2. Bai, B.; Esser, PD. The effect of edge artifacts on quantification of Positron Emission Tomography; Nuclear Science Symposium Conference Record (NSS/MIC), 2010 IEEE; 2010; p. 2263-2266.

3. Thielemans, K., et al. Impact of PSF modelling on the convergence rate and edge behaviour of EM images in PET; Nuclear Science Symposium Conference Record (NSS/MIC), 2010 IEEE; 2010; p. 3267-3272.

4. Snyder DL, et al. Noise and edge artifacts in maximum-likelihood reconstructions for emission tomography. IEEE Trans Med Imaging. 1987; 6(3):228-238. [PubMed: 18244025]

5. S Tong A, et al. Properties and Mitigation of Edge Artifacts in PSF-Based PET Reconstruction. IEEE Transactions on Nuclear Science. Oct; 2011 58(5):2264-2275.

6. Rapisarda E, et al. Evaluation of a New Regularization Prior for 3-D PET Reconstruction Including PSF Modeling. IEEE Transactions on Nuclear Science. Feb.2012 59(1) 
7. Yan M. EM-type algorithms for image reconstruction with background emission and Poisson noise. Advances in Visual Computing. 2011:33-42.

8. Shepp LA, Vardi Y. Maximum likelihood reconstruction for emission tomography. Medical Imaging, IEEE Transactions on. 1982; 1(2):113-122.

9. Thielemans K, et al. STIR: software for tomographic image reconstruction release 2. Physics in Medicine and Biology. Feb; 2012 57(4):867-883. [PubMed: 22290410]

10. Mourik JE, et al. Journal of Cerebral Blood Flow \& Metabolism. Oct; 2009 30(2):381-389. [PubMed: 19844240] 

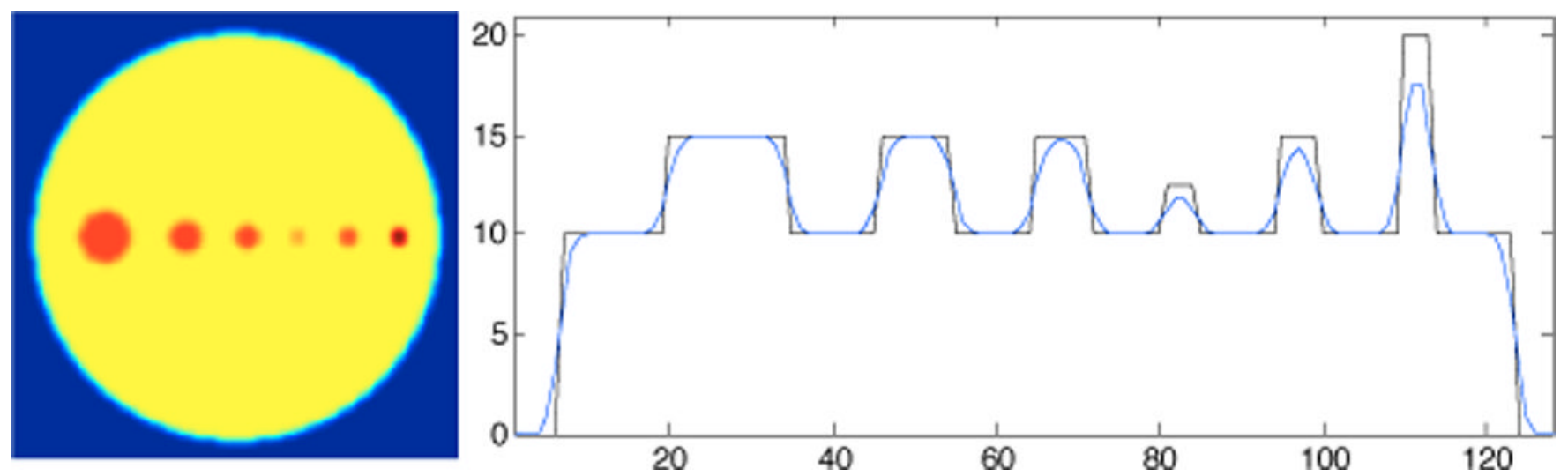

Figure 1.

Cylindrical phantom blurred with a $4.5 \mathrm{~mm}$ FWHM Gaussian kernel (left) and horizontal profile of pixel intensities through the midline (right) on: the actual phantom (blue line) and the ideal phantom (black line), shown for reference. 


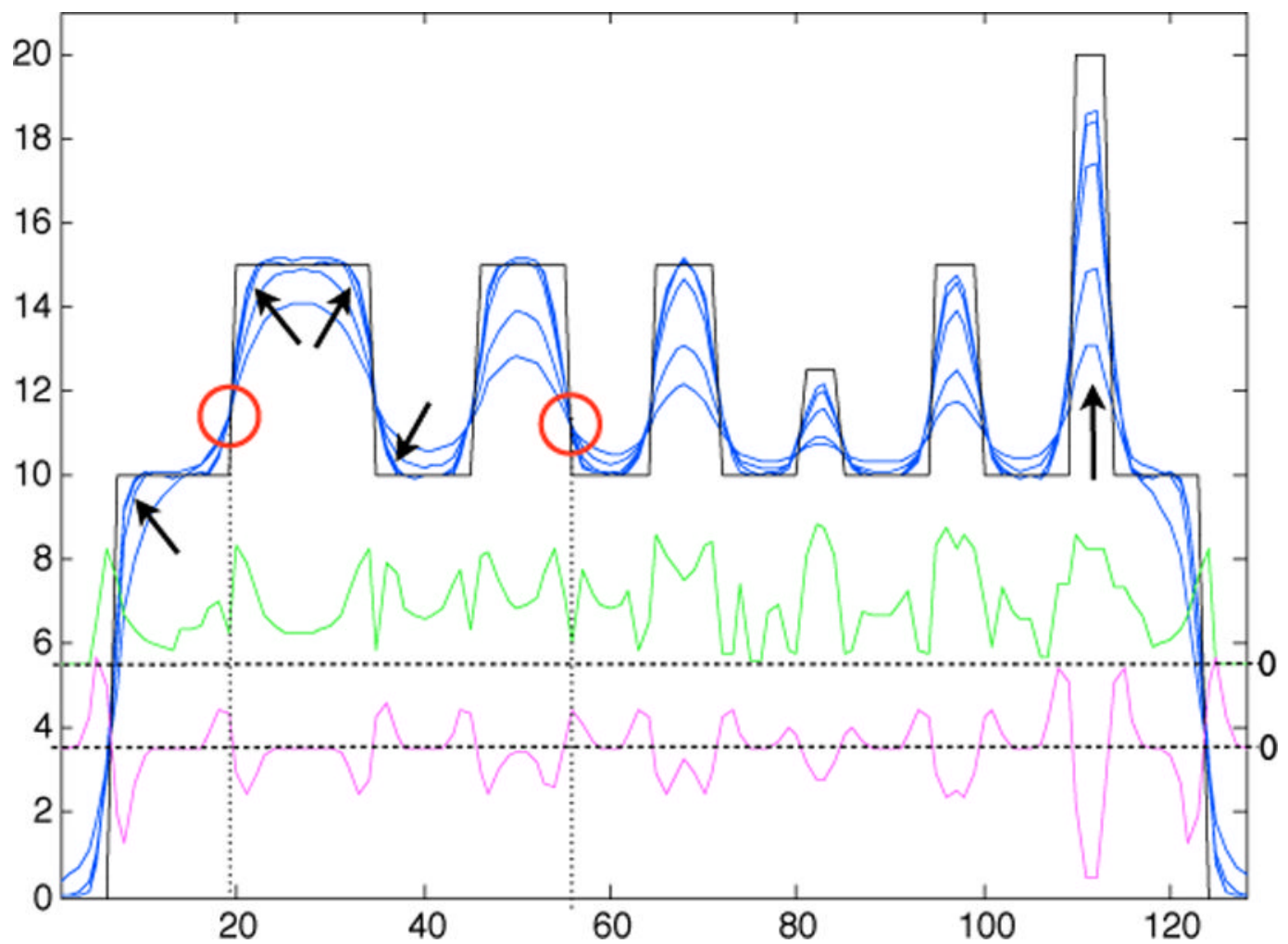

Figure 2.

Illustration of how $\lambda^{k}$ evolves and its relation with the image structure. Ideal phantom's horizontal midline profile (black line). Reconstructed (MLEM) profiles over several iterations of Eq. 1 (blue lines). Number of iterations to convergence (green line). Second derivative of the blurred phantom's profile (magenta line). Arrows show the dominant direction of evolution of reconstructed profiles over the iterations. Red circles highlight the localization of the inflection points of the reconstructed profiles, which take a relatively small number of iterations to converge. 


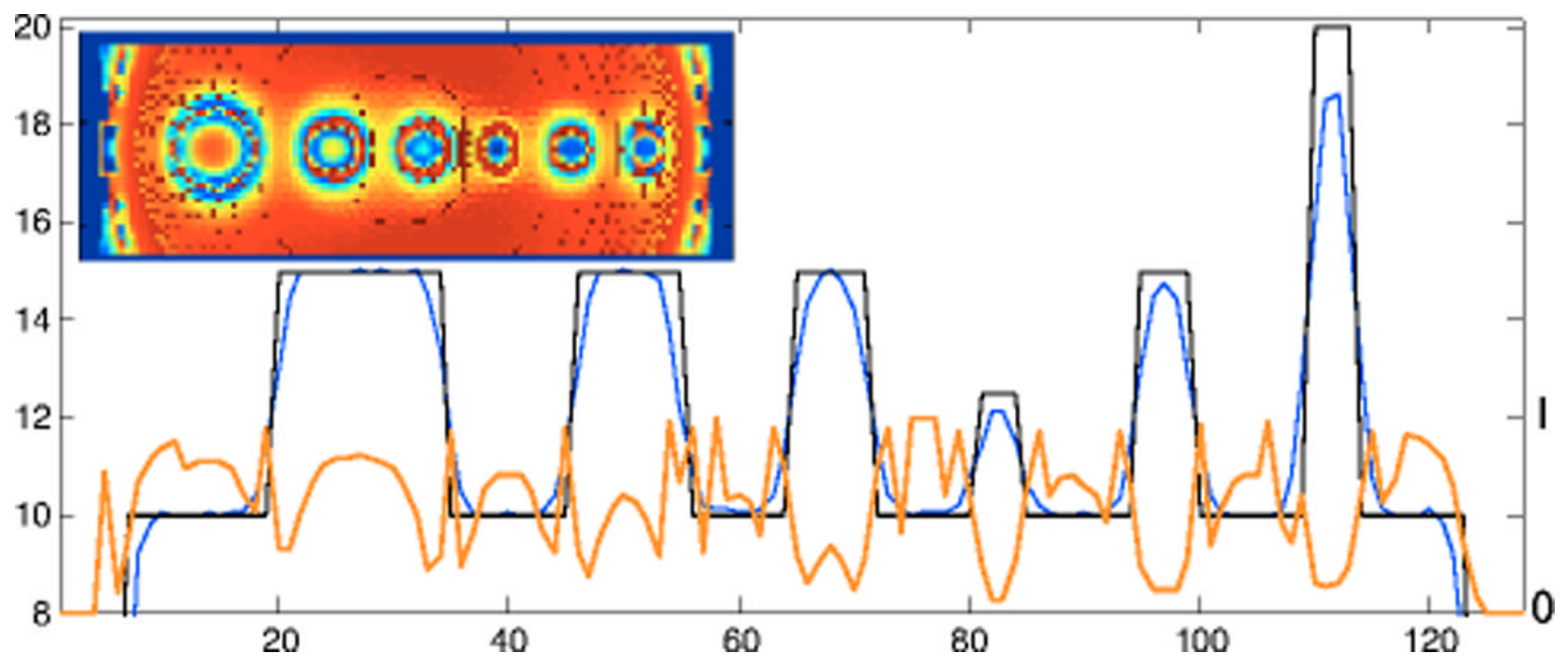

Figure 3 .

Horizontal profiles through the horizontal midline of ideal phantom (black line), MLEM reconstruction at convergence (blue line) and spatial TV weight $w_{b}$ (orange line), from the spatial weight map (top left). Left and right axes are for the profiles and $w_{b}$, respectfully. 


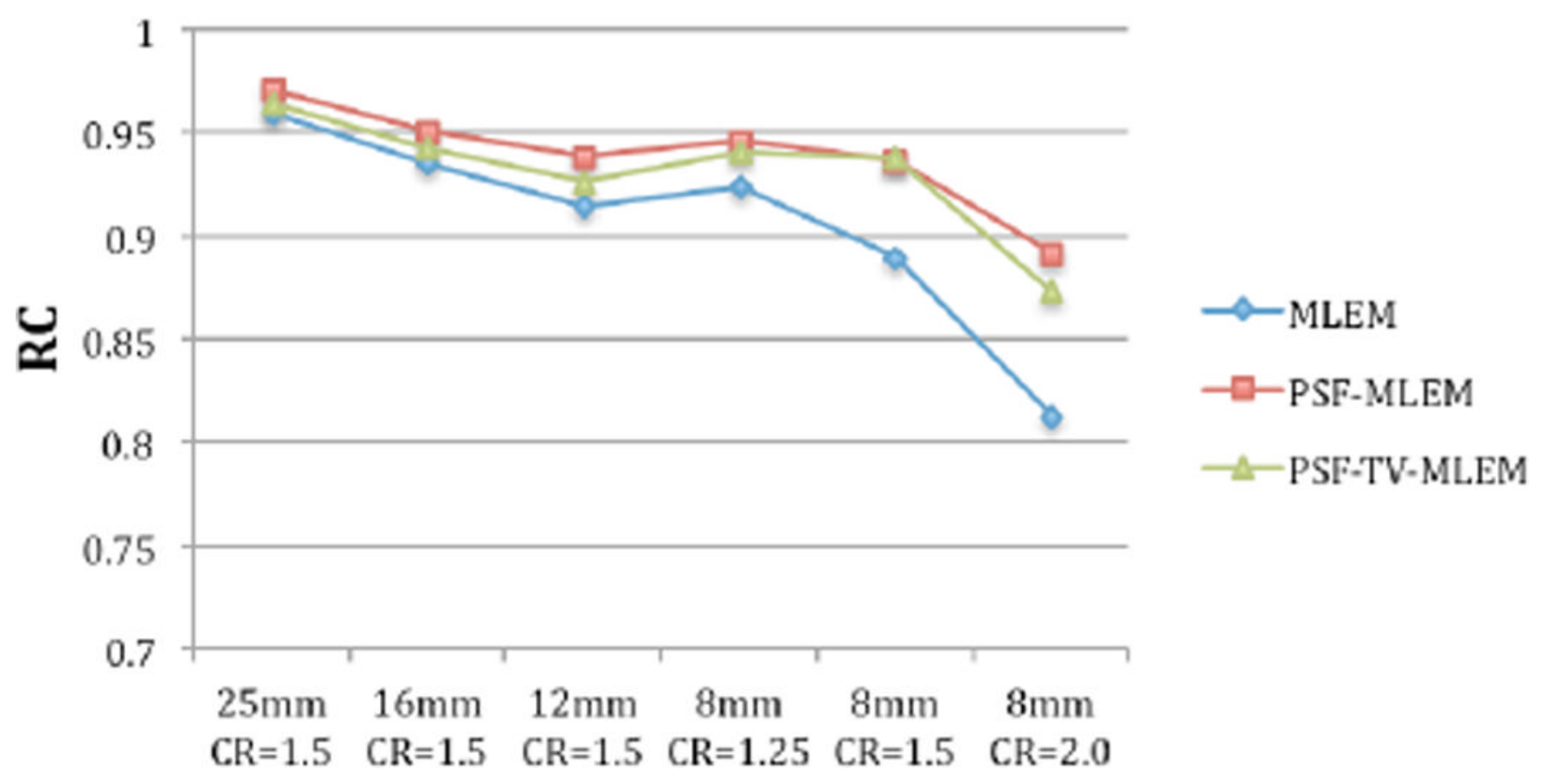

Figure 4.

Recovery coefficient (RC) measures for different size cylinders and contrast ratios (CR) of different reconstruction routines. The $x$-axis reports cylinder diameter $(\mathrm{mm})$ and contrast ratio $(\mathrm{CR})$ relative to the background. 


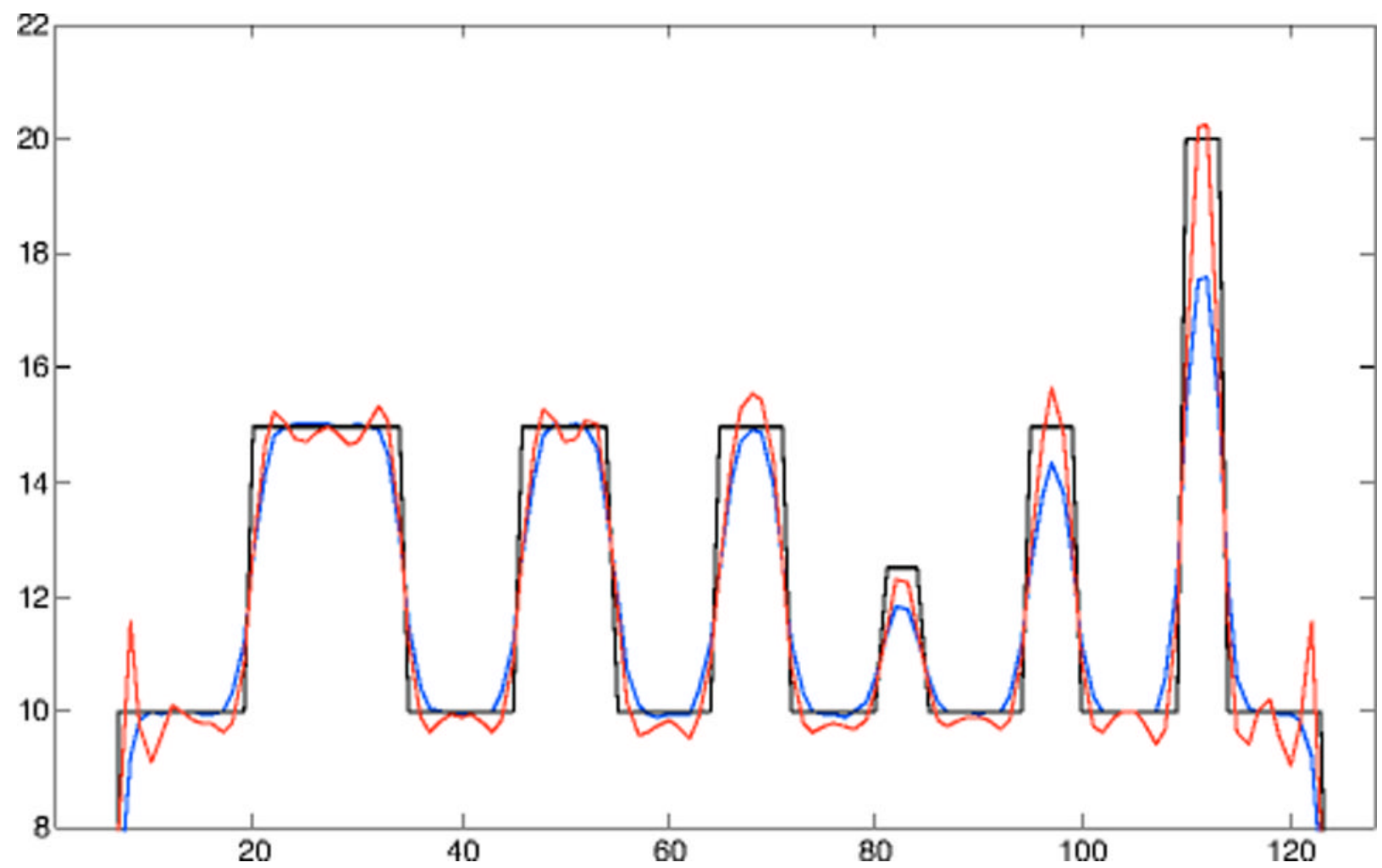

Figure 5.

Horizontal midline profile through the ideal (black line), MLEM (blue line) and PSF-MLEM (red line) reconstructions. 


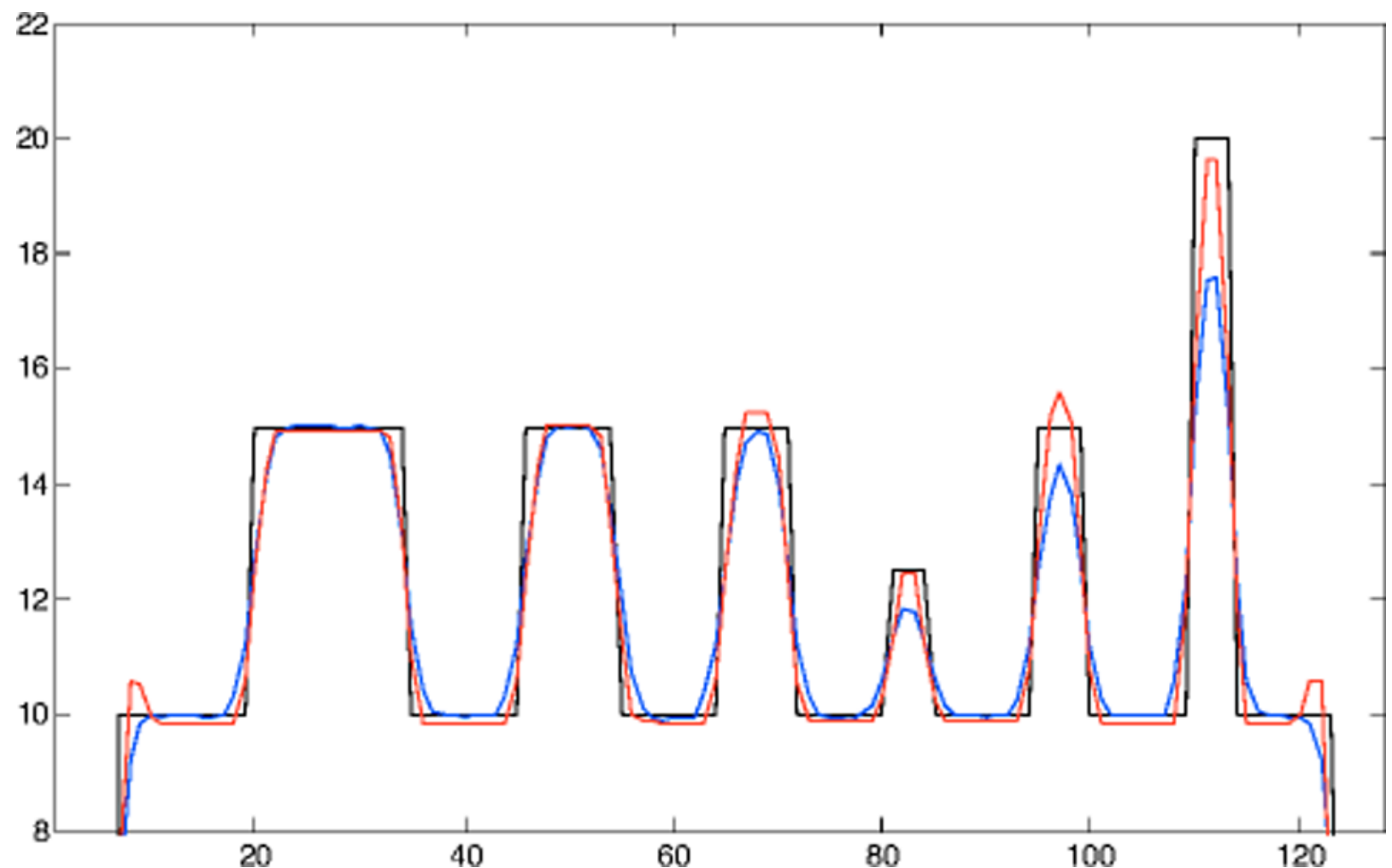

Figure 6.

Horizontal midline profile through the ideal (black line), MLEM (blue line) and TV-PSFMLEM (red line) with $\beta=0.02$ reconstructions. 

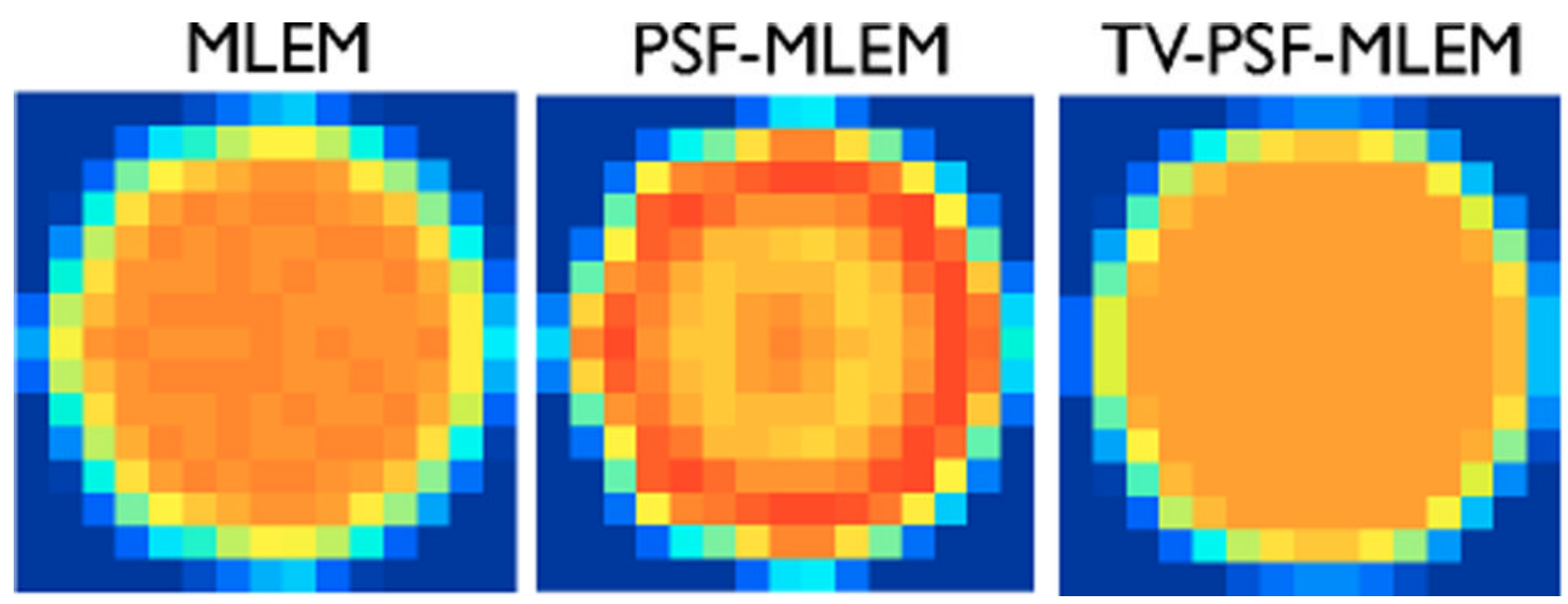

Figure 7.

Images of the $25 \mathrm{~mm}$ cylinder with three reconstructions (MLEM, PSF-MLEM, TV-PSFMLEM). TV-PSF-MLEM removes PSF-related ringing artifacts and restores homogeneity and contrast level that is similar to true phantom. 